\title{
INNOVATION UND EVALUATION. DIE PROGRAMMEVALUIERUNG DER CHRISTIAN DOPPLER LABORS UND JOSEF RESSEL ZENTREN
}

ROMAN HAAS, CHRISTIAN HELMENSTEIN, HERWIG W. SCHNEIDER

$\mathrm{W}$ as haben die beiden Begriffe Innovation und Evaluation gemein? Augenscheinlich zunächst den gleichen neuerdings progressiv verwendeten Suffix Ation. Laut Duden bezeichnet Ation in Bildungen mit Verben bzw. Verbstämmen wie innovare (lat. erneuern) oder valere (lat. bei Kräften sein, wert sein) das Ergebnis von einer Handlung oder Tätigkeit oder diese Handlung, Tätigkeit selbst. Beiden Termini eigen ist etwas Dynamisches wie Statisches, ein Prozess aber auch ein (abgeschlossenes) Ergebnis. Dieser philologische Blick entlarvt die aller Voraussicht nach mehrheitlich vorgenommene Begriffspaarung Innovation zu Dynamisch und Evaluation zu Statisch. Ein Vorurteil sei schon mal ausgeräumt. Alle vier Begriffe haben miteinander zu tun. Dynamisch und Statisch sind integrative Bestandteile der beiden Konzepte Innovation und Evaluation.

Im Jahr 2016 erhielt das Industriewissenschaftliche Institut, kurz IWI, gemeinsam mit dem Economica Institut für Wirtschaftsforschung den Auftrag die Christian Doppler Labors, kurz CD-Labors, und Josef Ressel Zentren, kurz JR-Zentren, zu evaluieren. Die beiden Institute erhielten bereits 2011 den Auftrag zur Programmevaluierung der Christian Doppler Labors, verstehen sich aber trotzdem auch weiter als Verfechter des Prinzips der Beratungsvielfalt. Denn Beratungsmonopole sind nicht nur ein Problem für den Steuerzahler, sondern stehen auch für Verkrustungen in Aussage und Struktur.

\section{TERMINUS INNOVATION ALS SYNONYM FÜR DIE CD- LABORS UND JR-ZENTREN}

Greifen wir zunächst den ersten Begriff des Titels auf: Innovation steht in diesem Artikel als Anspielung und Synonym für die CD-Labors und JR-Zentren. Und zwar im doppelten Sinn. Zum einen hinsichtlich ihres innovativen systemischen Ansatzes als Brückenglied zwischen Wirtschaft und Wissenschaft. Zum anderen hinsichtlich ihres Ziels, der Generierung innovativer Antworten auf unternehmerische Forschungsfragen. Eine Forschungsgruppe erarbeitet dabei Grundlagenwissen, das beim Unternehmenspartner/den Unternehmenspartnern in die Entwicklung neuer Produkte und Verfahren fließt. Dabei erfolgt ein reger Wissens- und Erfahrungsaustausch zwischen den Partnern (Spillover-Effekte).

Beide Institutionen, die CD-Labors wie JR-Zentren, sind vereint unter dem Dach der Christian Doppler Forschungsgesellschaft (CDG). Sie ist eine Wissenstransfer- bzw. Forschungseinrichtung zwischen Universitäten wie Fachhochschulen und der Industrie. Die Ziele der CDG sind die Förderung der anwendungsorientierten Grundlagenforschung in Österreich sowie die Nutzbarmachung des Wissenspotenzials der einschlägigen universitären Forschung für die industrielle Innovation und die Lösung von Industrieproblemen. Die CDG gilt in Österreich als Wegbereiterin für eine erfolgreiche Kooperation zwischen Wissenschaft und Wirtschaft. Konkret erfolgt dies in eigens eingerichteten Forschungseinheiten mit fixen Laufzeiten. CD-Labors an Universitäten und außeruniversitären Forschungseinrichtungen und seit 2012 auch JR-Zentren an Fachhochschulen. Ein besonderes Charakteristikum der CDG ist ihre Doppelrolle - einerseits als Förderagentur (Abwicklungsstelle), die über förderungsfähige Projekte entscheidet, und andererseits als gemeinnütziger Verein, der für die Einrichtung und den Betrieb der Forschungseinheiten (CD-Labors und JR-Zentren) verantwortlich ist.

\section{DIE STRUKTURMERKMALE UND PRINZIPIEN DER CDG}

Zu den Strukturmerkmalen der CDG zählen die Trägerschaft durch forschende Unternehmen, die Autonomie in allen wissenschaftlichen Angelegenheiten, die flexible, anpassungsfähige Struktur und die langjährige Erfahrung in der Kooperationskultur zwischen Wissenschaft und Wirtschaft. Die CDG hat sich den folgenden drei Prinzipien verschrieben, die sie zugleich charakterisieren: Thematische Offenheit, Flexibilität und Einbettung der Forschungseinheiten in bestehende Institutionen mit eigener Rechtspersönlichkeit. ${ }^{1}$ Mit der thematischen Offenheit soll gewährleistet werden, dass alle Themen, zu denen Unternehmen eine Fragestellung bzw. einen Forschungsbedarf haben, wichtig und zulässig sind (Bottom-up-Orientierung). Ausgestattet mit einem hohen Grad an Flexibilität können die Forschungseinheiten über ihre gesamte Laufzeit 
hinweg auf neue Entwicklungen reagieren, neue Module einrichten, neue Unternehmenspartner einbinden sowie das Budget anpassen. Das ist wesentlich, da gerade in der anwendungsorientierten Grundlagenforschung zu Beginn oftmals nur bedingt abgeschätzt werden kann, welche Ressourcen (z.B. in budgetärer Hinsicht) notwendig sind und welche Unternehmen bestenfalls eingebunden werden sollen. Somit kann gewährleistet werden, dass die im Laufe des Forschungsprozesses auftretenden und notwendigen konzeptionellen wie institutionellen Änderungen auch berücksichtigt werden können. Zweifellos erhöht sich damit die Erfolgswahrscheinlichkeit der Forschung. Flexible und anpassungsfähige Strukturen und Rahmenbedingungen braucht es nicht nur für die Generierung von Innovationen - auch nutzenbringende Evaluationen müssen sich dessen bedienen, dazu später mehr. Neben der thematischen Offenheit und der Flexibilität definiert die CDG die Einbettung der Forschungseinheiten in die institutionellen Strukturen der Universitäten, Fachhochschulen oder Forschungseinrichtungen als drittes wesentliches Prinzip. Bestehende Strukturen können so optimal genutzt und der notwendige bürokratische Aufwand auf ein Minimum reduziert werden, so das erklärte Ziel.

\section{DER AUFTRAG ZUR EVALUATION}

Der Dynamik der Zeit und/oder dem überproportional häufig auftretenden Verlangen nach einem steten "Rebranding" ist es geschuldet, dass sich der Name des Auftraggebers der vorliegenden Evaluation inzwischen geändert hat. Das ehemalige Bundesministerium für Wissenschaft, Forschung und Wirtschaft (BMWFW) - das jetzige Bundesministerium für Digitalisierung und Wirtschaftsstandort (BMDW) - hat die kombinierte Programmevaluierung der CD-Labors und JR-Zentren im Jahr 2016 in Auftrag gegeben. Evaluiert werden sollen die vom Wirtschaftsministerium lancierten und von der CDG abgewickelten Förderprogramme „Förderung der Einrichtung und des Betriebs von CR-Labors" und "Förderung und Einrichtung des Betriebs von JR-Zentren“. Zielsetzung ist es, das Förderprogramm bzw. die beiden zu evaluierenden Programme hinsichtlich ihrer Wirkungsebenen Output (Kennzahlenebene), Outcome (Ebene der operationalisierbaren Ziele) und Impact (Ebene der wirtschafts- und gesellschaftspolitischen Ziele) zu untersuchen. Der gesamte Evaluierungsprozess orientiert sich dabei am Vorbild der Nutzen-, Programm- und Systemevaluierung aus dem Jahr 2011 (Vorgängeruntersuchung), wodurch intertemporale Vergleiche möglich werden. Zusätzlich werden in der aktuellen Evaluierung die JR-Zentren - seit 2012 unter dem Dach der CDG - sowie ergänzende Module (Finanzierung der Grundlagenforschung, Patentanalyse) einbezogen.

\section{DIE INTERTEMPORALE EVALUATION EINES INNOVA- TIVEN SYSTEMS}

Evaluationsgegenstand der Analyse ist nicht eine Innovation per se, sondern evaluiert wird ein auf die Generierung von Innovationen ausgerichtetes System hinsichtlich seines Outputs. Output kann eine Innovation sein, die indirekt, z.B. in Form einer Patentanalyse, quantifiziert und so- mit evaluiert werden kann. Die Evaluation bedient sich dabei „statischer" Ergebnisse des Systems, z.B. in Form einer Kenndatenanalyse, um Rückschlüsse auf die Wirksamkeit des Systems in seiner dynamischen Form zu gewinnen. Definitorisch gesprochen, ist Evaluation demnach eine systematische Untersuchung und Bewertung eines Gegenstandes auf der Basis empirischer, d.h. erfahrungsbasierter Informationen, in der Absicht, seine Qualität und seinen Nutzen sicherzustellen und weiterzuentwickeln. ${ }^{2}$ Oder anders formuliert, zielt die Evaluation vordergründig auf eine statische Analyse für eine möglichst dynamische Weiterentwicklung eines Systems, in diesem Fall der CDG. Um die CDG und das Wirtschaftsministerium mit handlungsrelevanten Ergebnissen auszustatten, hat sich das Untersuchungsteam bestehend aus IWI und Economica vor allem den Fokus auf die Nutzungsorientierung auf die Fahnen geschrieben. Mit maßgeschneiderten Methoden soll der Gegenstand analysiert und nutzbringende Maßnahmenempfehlungen ausgearbeitet werden.

Ein wesentlicher Benefit der vorliegenden Evaluierung ist die direkte Vergleichbarkeit mit der Evaluation des Jahres 2011, die mit abgestimmten Methoden durchgeführt wurde. Dieser intertemporale Vergleich lässt spezifische Rückschlüsse auf Änderungen im Zeitraum 2011 bis 2016 zu, wodurch eruiert werden kann, welche Bereiche an Bedeutung zugenommen/verloren haben - wo z.B. Akzentuierungen im Vergleich zu 2011 stattgefunden haben - und inwiefern die damals empfohlenen Maßnahmen (bereits) umgesetzt wurden. Um derartige dynamische Vergleiche mit der notwendigen Reliabilität, Validität und Objektivität durchführen zu können, kann es einer gewissen „statischen" Verwendung von Methoden bedürfen. Hier kann ein gewisser Zielkonflikt mit dem Einsatz möglichst aktueller, neuentwickelter Methoden festgemacht werden.

Neben diesem zeitlich verschobenen Bezugsfeld werden in der vorliegenden Evaluierung auch systemische Vergleiche für Evaluationen herangezogen (Stichwort Benchmark). So liegt es hinsichtlich des vorliegenden Evaluationsgegenstandes auf der Hand die CD-Labors mit den JR-Zentren zu vergleichen. Dabei können CD-Labors als Bezugsfeld für die JR-Zentren dienen und umgekehrt. Durch derartige systemische Gegenüberstellungen können relative charakteristische Besonderheiten abgeleitet werden, die wiederum Hilfe bei der Ergebnisinterpretation liefern. Aufgrund der noch relativ frühen Entwicklungsphase der JR-Zentren sind derartige intraorganisatorische Vergleiche freilich mit Vorsicht zu analysieren. Für den intertemporalen Vergleich sind die JR-Zentren überhaupt ausgeschlossen, da sie in der Vorgängeruntersuchung noch nicht berücksichtigt werden konnten.

\section{DIE WICHTIGSTEN ERGEBNISSE DER EVALUATION}

Der intertemporale wie intraorganisatorische Vergleich nimmt den wichtigsten Analyseteil der gegenständlichen Evaluation ein. So konnte im Rahmen der aktuellen Kenndatenerhebung sowie einer Programmund Systemevaluierung beispielsweise festgestellt werden, dass der Output je CD-Labor und je Million Laborbudget bei einigen Kennzahlen wie der Anzahl der Habilitationen, der Anzahl der Publikationen und der Konferenzbeiträge im Vergleich zur letzten Evaluierung angestiegen ist. Demgegenüber steht ein Rückgang bei der Anzahl der Dissertationen, 
der Preise bzw. Rufe und der Patente. Generell geht mit einer Erhöhung des CD-Laborbudgets auch ein Anstieg der Outputs einher.

Das CD-Laborprogramm überzeugt nach wie vor durch hohe Flexibilität und Anpassungsfähigkeit und weist auch nach Jahrzehnten des Bestehens einen hohen Erfolg im Bereich der Wirtschafts-Wissenschafts-Kooperationsbeziehungen auf, so ein weiteres Ergebnis. Es ist ein Förderinstrument mit klarem Förderkonzept und -ziel sowie hohem Zielerreichungsgrad und „eigenem Charakter". Vor allem die Langfristigkeit (inkl. Planbarkeit) und die dadurch ermöglichte intensive Kooperation bilden einen starken (Forschungs-)Kern, und sorgen damit für einen nachhaltigen Humankapitalaufbau. Das CD-Labor ist somit nicht nur ein weiteres Förderprogramm, es formt eine intensiv und fokussiert arbeitende (Forschungs-) Gruppe aus. Als renommiertes, historisch gewachsenes Förderprogramm erzeugt es auch Sichtbarkeit. CD-Labors werden als Marke über die österreichischen Grenzen hinaus wahrgenommen und stärken dadurch auch den heimischen Forschungsstandort.

Einfache Stakeholder Strukturen sowie die zielorientierte und gute Zusammenarbeit der CDG mit dem Wirtschaftsministerium als Programmträger führen zu einer hohen Qualität in der Programmgestaltung sowie zu einer effizienten Programmabwicklung, so ein erstes Resümee.

Mittels empirischer Erhebung (Tiefeninterviews und Online-Befragung) konnte ferner festgestellt werden, dass die Zufriedenheit der Fördernehmer (Wissenschaft, Wirtschaft) mit dem Programm nach wie vor sehr hoch ist. Dies betrifft insbesondere auch die (Betreuungs-)Leistungen des CDG Generalsekretariats. Die bei der letzten Evaluierung (2011) sowie bei der Studie zu Handlungsoptionen für eine weitere Optimierung des CD-Programms (2013) angeregten Empfehlungen wurden von Seiten der Programmverantwortlichen (weitestgehend) aufgenommen, und die von der CDG umgesetzten Maßnahmen vor allem hinsichtlich administrativer Vereinfachungen zeigen bereits positive Wirkungen. Verbesserungspotential im Schnittstellenmanagement besteht aber in einzelnen Fällen. Zwischen Universität(sverwaltung) und CDG konnten teils Reibungsverluste identifiziert werden, wodurch vermeidbarer administrativer Aufwand für die beteiligten Laborleiterlnnen entsteht. Hier gibt es Ansatzpunkte die bereits durchgeführten Verbesserungen noch fortzuführen.

Für die Partnerunternehmen aus der Wirtschaft bietet das CD-Programm ein breites Nutzenspektrum, so ein weiteres wesentliches Ergebnis. Dieses reicht von der Bewertung verschiedener Lösungsstrategien (Radarfunktion) über die inkrementelle Verbesserung bestehender Produkte/Prozesse bis hin zur Entwicklung neuer Produktionsverfahren und radikal neuer Produkte. Hohen Nutzen durch die Beteiligung an einem CD-Labor sehen die Unternehmen vor allem im Kompetenzaufbau, dem Aufzeigen neuer technologischer Optionen und in der Stärkung ihrer technologischen Problemlösungskompetenz. Positiv ist weiters anzumerken, dass verbunden mit dem Wissenstransfer eine Erhöhung der Wettbewerbsfähigkeit gegenüber Konkurrenten verbunden mit einer Stärkung des Unternehmensstandorts einhergeht.

Die JR-Zentren, als Pilotprojekt bereits 2008 ins Leben gerufen, wurden 2012 inhaltlich und organisatorisch an die CDG angekoppelt. Wesentliche Elemente des CD-Modells wurden dabei übernommen und in adaptierter Form auf das JR-Zentren-Programm übertragen. Im Rahmen der Evaluierung konnte eine geringe Zahl an laufenden JR-Zentren be- trachtet werden, denen - ähnlich den CD-Labors - ein erster positiver Evaluierungsbefund ausgestellt werden kann. Die JR-Zentren befinden sich aber noch in einer frühen Entwicklungsphase. Es bleibt abzuwarten, ob zukünftige Evaluierungen Änderungsbedarf beim Programmdesign sehen, oder ob ein eigener Charakter, vor allem gegenüber den CDLabors, bereits mit der derzeitigen Ausgestaltung des Förderprogramms erkennbar sein wird. Dies muss auch im Einklang und unter Berücksichtigung zukünftiger Rahmenbedingungen der Fachhochschulen bzw. des Fachhochschulwesens erfolgen. Eine systemische Relevanz der JR-Zentren im Nationalen Innovationssystem (NIS) wird erst in den kommenden Jahren zu beurteilen sein.

\section{EIN MULTIMETHODISCHER ANALYSEANSATZ}

Das Studienteam hat zur Evaluation der Untersuchungsobjekte einen multimethodischen Ansatz gewählt. Sowohl quantitative wie qualitative Methoden der empirischen Sozialforschung (wie Primär- und Sekundäranalysen) werden eingesetzt, um die CD-Labors und JR-Zentren holistisch zu untersuchen. Neben dem bereits erörterten intertemporalen und intraorganisatorischen Vergleich, kommen auch mehrperspektivische Untersuchungen (Teilgruppenanalysen) zum Einsatz.

Die vorgenommene Kenndatenerhebung, in der Evaluierung 2011 noch Nutzenevaluierung genannt, dient der Erfassung des Zielerreichungsgrades auf der Ebene der 45 analysierten CD-Labors und 6 anaIysierten JR-Zentren. Mittels dieser quantitativen Methode wird der Output der Forschungseinrichtungen erhoben und dargestellt sowie in Relation zum Input gesetzt. Ohne Bezugsrahmen lässt diese Methode lediglich deskriptive Aussagen zu. Durch den intertemporalem Vergleich mit der Nutzenevaluierung des Jahres 2011 bekommen die statischen Ergebnisse ihren eigentlichen Wert, wodurch nicht nur einfache Beschreibungen, sondern auch weiterführende dynamische, ja explorative Interpretationen angestellt werden können. Im Zuge der Kenndatenerhebung spielte auch die Messung von Additionalitätswirkungen eine besondere Rolle, durch den jener Output gemessen wird, der ohne Förderung nicht zustande gekommen wäre.

Neben der Kenndatenerhebung wird eine Programm- und Systemevaluierung auf Basis von qualitativen Erhebungen (Online-Erhebung, Tiefeninterviews) durchgeführt. Hierbei wird auf die unterschiedlichen Perspektiven, in der Gestalt der einzelnen Stakeholder des CDG-Programms (Wirtschaftsministerium, CDG, Wirtschaft, Wissenschaft), besonders Acht gegeben. Um etwaige unterschiedliche Präferenzen hinsichtlich der operationalen Ziele der einzelnen Stakeholder identifizieren zu können, kam der Analytische Hierarchische Prozess (AHP) zur Anwendung. ${ }^{3}$

Die erweiterte Systemevaluierung befasst sich mit der Analyse der Unternehmensbeteiligungen an den CD-Labors und JR-Zentren im Laufe der Zeit und einer Patentanalyse. Mit ersterem kann die Dynamik in der Integration von neuen Unternehmen in die CD-Labors bzw. JR-Zentren untersucht werden, mit zweiterem können die nationalen Technologiestärkefelder identifiziert werden. Durch die Verschränkung dieser beiden Analysemethoden kann eruiert werden, welche Unternehmen in der Vergangenheit durch das Förderprogramm unterstützt wurden, die auf 
Grund der Förderung dazu beigetragen haben, die entwickelte Technologie als nationales Stärkefeld zu etablieren.

Der multivariate Analyseansatz hat eine Vielzahl an Ergebnissen hervor gebracht, die zu einem 288-seitigen Studienbericht verdichtet wurden. Der gewählte Ansatz, einer relativ statischen Evaluation eines flexiblen, offenen und dynamischen Systems hat sich als richtig erwiesen, da eben gerade dieser statische Ansatz eine dynamische Analyse ermöglicht.

\section{DIE DYNAMIK DER CDG ALS INNOVATIVES SYSTEM}

So innovativ, flexibel und offen ein System auch konzipiert ist, ist es nie davor gefeit, an Dynamik und Kreativität zu verlieren und zu einem statischen Gebilde zu verkommen. Es muss jedenfalls proaktiv daran gearbeitet werden, dass sich das System immer wieder von selbst erneuert. Ein System, das Innovationen hervorbringen soll, muss auch selbst innovativ/dynamisch sein. Ähnlich der Schumpeter’schen Idee der schöpferischen Zerstörung, müssen Neuentwicklungen auch dazu führen dürfen, dass alte Strukturen aufgelöst werden. Die Integration der JR-Zentren in die CDG kann durchaus eine schöpferische Wirkung auch für die CD-Labors selbst haben. Da davon ausgegangen werden kann, dass der Zugang neuer Unternehmen über die JR-Zentren leichter fällt. Essentiell wird es jedenfalls sein, dass neue Unternehmen (Startups etc.) in die CDG-Landschaft aufgenommen werden. Junge Unternehmen haben Potential mit ihren Ideen und neuen Herangehensweisen dem System immer wieder frischen Wind zu verleihen. Letztlich lebt das ganze System von neuen Ideen, von neuen Innovationen, die bestenfalls auch Marktreife erlangen.

\section{DIE DYNAMIK DER EVALUATION}

Die Forderung nach stetiger Dynamik und Agilität richtet sich nicht nur an die CDG, sondern auch an den Prozess der Evaluation. Bei der Auswahl bzw. Mischung bestehender und der Konzipierung neuer Evaluationsmethoden ist innovatives und kreatives Potenzial gefragt. Der innovative und kreative Aspekt entspringt dabei nicht der Methode selbst, sondern resultiert aus der Anwendung der Methode am Untersuchungsobjekt. Innovativ meint in diesem Zusammenhang die Auswahl bzw. Neukonzipierung der für den jeweiligen Untersuchungsgegenstand zweckdienlichsten Methoden. Das Feld der Möglichkeiten ist hier - vor allem auch in der industriellen Forschung - beinahe unbegrenzt. Gedankengrenzen sind nicht angebracht, vielmehr ist "Out-of-the-Box" Denken gefragt. Man sieht, Evaluation und Innovation, sind sich näher als gedacht.

Titel der Studie:

Kombinierte Programmevaluierung der Christian Doppler Labors und Josef Ressel Zentren 2016

\section{AUTORINNEN DER STUDIE}

\author{
RAIMUND ALT \\ HELMUT BERRER \\ JULIA BORRMANN \\ PHILIPP BRUNNER \\ BERNADETTE DOLLE \\ CHRISTIAN HELMENSTEIN \\ JASMIN JÖCHLE \\ JOHANNA PIRKER \\ PAULINE POHL \\ JONAS POPKO \\ MATTHIAS SCHMIDL \\ HERWIG W. SCHNEIDER
}

Economica, Institut für Wirtschaftsforschung

Liniengasse 50-52, 1060 Wien

www.economica.at

IWI, Industriewissenschaftliches Institut

Mittersteig 10/4, 1050 Wien

$\underline{\text { www.iwi.ac.at }}$ 